\title{
Brightness temperature for radio sources
}

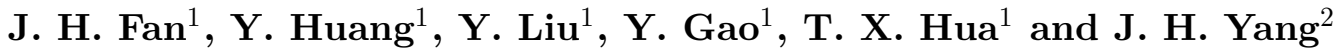 \\ ${ }^{1}$ Center for Astrophysics, Guangzhou University, Guangzhou 510006, China \\ ${ }^{2}$ Dept of Phys and Electron. Science, Hunan Univ. of Arts and Sci., Changde, China
}

\begin{abstract}
In this work, using the database of the university of Michigan Radio Astronomy Observatory (UMRAO), we determined the brightness temperatures, $\mathrm{T}_{\mathrm{B}}$ for a sample of 167 radio sources. The value of $\mathrm{T}_{\mathrm{B}}$ is in a range of $\log \mathrm{T}_{\mathrm{B}}(\mathrm{K})=10.46$ to 20.08 , which suggested that the boosting factors are in a range of $\delta=0.41$ to 41.26 .
\end{abstract}

Keywords. galaxies: active, methods: data analysis, galaxies: jets

\section{Introduction}

Blazars form an extreme subclass of Active Galactic Nuclei (AGNs), showing extremely observational properties(e.g. Aller et al. 2003; Cellone et al. 2007; Efimov et al. 2002; Fan, 2005). These extremely observational properties are associated with the beaming effect. Up to now, boosting factors are only available for large samples in the papers by Ghisellini et al. (1993)-(G93) and Lahteenmark \& Valtaoja, (1999)-(LV99). Therefore, we used the UMRAO database for the determination of boosting factor for a larger sample.

\section{Brightness Temperature and Boosting Factor}

From the work(Wagner \& Witzel, 1995), the brightness temperature can be calculated by $\mathrm{T}_{\mathrm{B}}=\left(4.5 \times 10^{10} \mathrm{~K}\right) \mathrm{F}\left[\frac{\lambda \mathrm{d}}{\mathrm{t}_{\mathrm{obs}}(1+\mathrm{z})}\right]^{2}$, here $\mathrm{F}$ is the flux density in Jy, $\lambda$ the wavelength in $\mathrm{cm}$, $\mathrm{d}$ the distance in Mpc, and $\mathrm{t}_{\mathrm{obs}}$ the time scale in days. From the UMRAO database, we calculated the brightness temperature $\mathrm{T}_{\mathrm{B}}$ for 167 sources. The obtained temperatures are in the range of $\log T_{B}=10.46$ to 20.08 . If the brightness temperature is explained as the boosting effect as argued by Qian et al. (1991) and Romero et al. (1994), we can estimate the boosting factors are in the range of $\delta=0.41 \sim 41.26$. This results are consistent with the range of $\delta$ by G93 and LV99.

\section{Acknowledgements}

This work is supported by NSFC(10573005,10633010) and the 973 project(2007CB815405). This research has made use of data from the UMRAO which has been supported by the University of Michigan and the National Science Foundation.

\section{References}

Aller, M. F., Aller, H. D., \& Hughes, P. A. 2003, ApJ, 586, 33

Cellone, S. A., Romero, G. E., \& Araudo, A. T. 2007, MNRAS, 374, 357

Efimov, Y. S., Shakhovskoy, N. M., Takalo, L. O., \& Sillanpaa, A. 2002, A\&A, 381, 408,

Ghisellini, G., Padovani, P., Celotti, A., \& Maraschi, L. 1993, ApJ 407, 65

Lahteenmark, L. \& Valtaoja, E. 1999, ApJ, 521, 493

Qian, S. J., Quirrenbach, A., Witzel, A., et al. 1991, A\&A, 241, 15

Romero, C. E., Combi, J. A., \& Colomb, F. R. 1994, A\&A, 288, 731

Wagner, S. \& Witzel, A. 1995, ARA\&A, 33, 163 
Table. Brightness temperature and Boosting factor of radio sources

\begin{tabular}{|c|c|c|c|c|c|c|c|c|}
\hline Narne & $\log T_{B}$ & $\stackrel{s}{e}$ & Name & $\log \mathrm{T}_{3}$ & $\delta$ & Narne & $\log _{B}$ & $\delta$ \\
\hline $0003-066$ & 16.57 & 8.21 & $0818-128$ & 17.63 & 13.35 & $1606+106$ & 18.06 & 16.31 \\
\hline $0007+106$ & 17.01 & 10.04 & $0829+046$ & 16.67 & 8.59 & $1600+660$ & 16.70 & 8.72 \\
\hline $001.6+731$ & 17.35 & 11.75 & $0881+55 ?$ & 12.58 & 1.28 & $1611+343$ & 18.00 & 15.86 \\
\hline $0022+538$ & 15.91 & 6.05 & $0886+710$ & 18.65 & 21.42 & $164+416$ & 18.24 & 17.78 \\
\hline $0040+517$ & 15.11 & 4.18 & $0838+133$ & 17.94 & 15.40 & $1633+382$ & 17.73 & 14.00 \\
\hline $0048-097$ & 17.38 & 11.90 & $0850+581$ & 16.27 & $7.1 \mathrm{E}$ & $1634+628$ & 17.05 & 10.25 \\
\hline $0050+581$ & 17.32 & 1.1 .58 & $0851+202$ & 16.82 & 0.10 & $1637+574$ & 17.54 & 12.81 \\
\hline $0106+013$ & 18.28 & 1.7.60 & $0850+470$ & 16.53 & 8.07 & $16 \div 1+309$ & 17.36 & 11.83 \\
\hline $0108+388$ & 16.81 & 0.30 & $0906+430$ & 17.38 & 11.90 & $1642+600$ & 18.10 & 16.50 \\
\hline $0109+224$ & 17.54 & 12.84 & $0912+297$ & 16.20 & 7.22 & $1652+908$ & 15.33 & 4.69 \\
\hline $0127+233$ & 16.98 & 8.80 & $0017+458$ & 15.21 & 4.38 & $1717+178$ & 16.70 & 0.07 \\
\hline $0139+476$ & 17.74 & 1.4 .00 & $0917+624$ & 18.18 & 16.82 & $1721+343$ & 15.68 & 5.44 \\
\hline $0134+320$ & 16.18 & 6.85 & $0923+392$ & 16.91 & 9.59 & $1727+502$ & 16.07 & 6.53 \\
\hline $0153+744$ & 16.84 & 0.28 & $0951+690$ & 10.47 & 0.49 & $1730-130$ & 17.48 & 12.45 \\
\hline $0202+140$ & 1.7 .19 & 10.90 & $095 \dot{i}+556$ & 17.41 & 12.05 & $1741 \ldots 038$ & 18.21 & 17.48 \\
\hline $0212+755$ & 17.37 & 11.87 & $0954+658$ & 17.63 & 13.34 & $1749+006$ & 17.52 & 12.71 \\
\hline $0215+015$ & 17.80 & 14.49 & $0957+227$ & 16.97 & 9.85 & $3+701$ & 18.58 & 20.74 \\
\hline $0218+357$ & 17.45 & 12.32 & $1003+351$ & 15.55 & 5.13 & $1803+78.4$ & 18.03 & $16.0 \%$ \\
\hline $0219+428$ & 16.83 & 0.25 & $1081+557$ & 16.70 & 8.71 & $1807+698$ & 15.01 & 4.00 \\
\hline $0220+427$ & 13.48 & 1.08 & 1084.208 & 16.84 & 0.20 & $1828+568$ & 17.61 & 18.26 \\
\hline $0234+285$ & 17.73 & 14.00 & $1038+528$ & 18.71 & 22.00 & $1828+48 \%$ & 17.81 & 14.50 \\
\hline $0235+164$ & 18.30 & 18.05 & $1040+123$ & 17.87 & 14.80 & $1842+455$ & 14.58 & 3.27 \\
\hline $0800+470$ & $16.3 ?$ & 7.48 & $1055+018$ & 18.25 & 17.75 & $1845+797$ & 15.04 & 1.05 \\
\hline $0806+102$ & 17.35 & 1.1.?5 & $1100+772$ & 16.32 & 7.30 & +319 & 17.17 & 10.70 \\
\hline $0815+416$ & 13.65 & 2.14 & $1101+384$ & 15.41 & 4.80 & 192 & 17.67 & 13.64 \\
\hline $0316+413$ & 10.41 & 0.48 & $1127-145$ & 17.71 & 13.80 & $1928+738$ & 17.32 & $11.5 ?$ \\
\hline $0323+022$ & 17.06 & 10.27 & $1139+704$ & 15.71 & 5.52 & $1989+605$ & 16.14 & 6.72 \\
\hline $0333+321$ & 17.78 & 1.4 .34 & $1137+660$ & 16.12 & 5.65 & $1951+488$ & 18.72 & 22.11 \\
\hline $0336-019$ & 17.84 & 15.30 & $1147+245$ & 16.62 & 8.38 & $1954+513$ & 17.78 & 14.32 \\
\hline $0355+508$ & 10.27 & 28.40 & 1148001 & 16.05 & 6.46 & $2005+403$ & 18.09 & 16.50 \\
\hline $0404+768$ & 16.24 & 7.05 & $1150+295$ & $17.9 ?$ & 15.61 & $2007+777$ & 11.02 & 11.07 \\
\hline $0420-014$ & 18.29 & 18.10 & $1157+732$ & 15.95 & 0.17 & $2014+$ & 16.44 & 7.74 \\
\hline $0422+004$ & 17.70 & 14.40 & $1215+903$ & $16.8 \%$ & 9.40 & $2020+614$ & 15.69 & 5.47 \\
\hline $0480+052$ & 14.86 & 3.73 & $121 \gamma+023$ & 15.54 & 5.11 & $2032+107$ & 17.60 & 13.21 \\
\hline $0440-003$ & 17.51 & 12.64 & $1219+285$ & 16.50 & 8.27 & $2121+053$ & 18.27 & 17.98 \\
\hline $0454-234$ & 17.15 & 10.72 & $1222+216$ & $1 ? .5 ?$ & 1.3 .01 & 2131-021 & 17.33 & 11.65 \\
\hline $0456-020$ & 18.25 & 17.81 & $1225+206$ & 18.46 & 19.62 & $2134+004$ & $17.8 \%$ & 14.92 \\
\hline $0518+165$ & 18.00 & 16.51 & $1226+023$ & 13.32 & 1.84 & $2136+141$ & 17.21 & 11.00 \\
\hline $0521-365$ & 14.98 & 3.95 & 1253055 & 16.32 & 7.80 & $2145+067$ & 17.80 & 15.05 \\
\hline $0528+134$ & 18.11 & 16.64 & $1254+476$ & 16.63 & 8.44 & 877 & 17.20 & 10.97 \\
\hline $0528-250$ & 18.88 & 23.82 & $1307+121$ & 17.56 & 12.97 & 215 & 17.62 & 13.32 \\
\hline $0538+498$ & 16.58 & 8.25 & $1308+326$ & 17.71 & 13.88 & 215 & 16.09 & 9.86 \\
\hline $2+308$ & 17.40 & 12.00 & $1328+307$ & 17.08 & 10.38 & $2200+420$ & 17.17 & 10.81 \\
\hline $0605+480$ & 15.57 & 5.18 & $1335-127$ & 17.48 & 12.46 & $2202+315$ & 17.62 & 13.29 \\
\hline $060 \mathrm{E}-08 \mathrm{E}$ & 17.64 & 13.43 & $1354-152$ & 18.00 & 15.86 & $2223-052$ & 17.09 & 15.75 \\
\hline $0607-157$ & 17.24 & 11.10 & $1358+624$ & 17.08 & 10.35 & $2220+901$ & 14.60 & 3.32 \\
\hline $0710+430$ & 16.60 & 8.82 & $1.400+162$ & 16.95 & 0.78 & +114 & 17.30 & 11.78 \\
\hline $0711+356$ & 18.90 & 18.18 & $1409+524$ & 15.17 & 4.31 & $2243+394$ & 16.27 & 7.15 \\
\hline $0716+714$ & 20.08 & 41.26 & $1413+135$ & 16.88 & 9.45 & $2251+158$ & 17.56 & 12.94 \\
\hline $0723+670$ & 17.84 & 14.71 & $1418+546$ & 15.66 & 5.40 & $2254+074$ & 16.06 & 6.48 \\
\hline $0785+178$ & 19.38 & 29.90 & $1.458+71.8$ & 16.08 & 6.55 & $2311+612$ & 16.40 & 7.02 \\
\hline $0754+100$ & 16.87 & 9.42 & $1504-166$ & 16.32 & 7.31 & $2335+081$ & 16.86 & 0.98 \\
\hline $0804+490$ & 18.46 & 19.50 & $1510-080$ & 16.96 & 9.80 & $2345-167$ & 17.50 & 12.50 \\
\hline $0808+010$ & 17.40 & 12.52 & $1514+197$ & 18.32 & 18.38 & $2351+156$ & 18.39 & 10.01 \\
\hline $0809+\frac{183}{8}$ & 19.12 & 26.40 & $1588+148$ & 17.06 & 10.28 & $2352+495$ & 15.81 & $6.0 ?$ \\
\hline $081 \frac{1}{4}+\frac{425}{4}$ & 16.95 & 9.78 & $1543+005$ & 17.57 & 13.00 & & & \\
\hline
\end{tabular}

